\title{
Valoración del uso de las nuevas tecnologías en personas con esclerosis múltiple
}

\section{Assessment of the use of new technologies in people with multiple sclerosis}

\begin{abstract}
Resumen
Este trabajo comprueba la importancia de la aplicación de las nuevas tecnologías unidas a la actividad física en la rehabilitación de personas con discapacidad. En la intervención se ha llevado a cabo un entrenamiento del equilibrio y la coordinación con una duración de seis semanas, usando la plataforma NeuroAthome en personas con esclerosis múltiple. Se han podido comprobar las mejoras en algunos de los parámetros medidos y, por tanto, promover el uso de las nuevas tecnologías para la rehabilitación de este colectivo.
\end{abstract}

\section{Palabras clave}

Esclerosis múltiple, EM, equilibrio, coordinación, NeuroAthome.

\begin{abstract}
This study shows the importance of the application of new technologies, together with the physical activity in the rehabilitation of people with disabilities. In the intervention, a training on balance and coordination has been carried out during six weeks, using the NeuroAthome platform in people with multiple sclerosis. This enabled us to check the improvements in some of the measured parameters and, therefore, to promote the use of new technologies for the rehabilitation of those group.
\end{abstract}

\section{Keywords}

Multiple sclerosis, MS, balance, coordination, NeuroAthome.

\author{
Marta Cárdenas Martínez \\ $<$ martacm.23@hotmail.com> \\ Universidad de Castilla-La Mancha. \\ España
}

\section{Marta Torres Parejo}

$<$ marta_torres_39@hotmail.com>

Universidad de Castilla-La Mancha. España

\section{Nuria Mendoza Laiz \\ <nuria.mendoza@ufv.es>}

Universidad Francisco de Vitoria. España

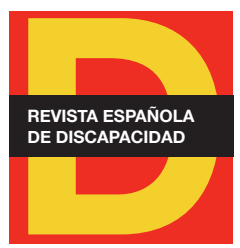

Para citar:

Cárdenas, M. et al. (2018):

"Valoración del uso de las nuevas tecnologías en personas con esclerosis múltiple". Revista Española de Discapacidad, 6 (II): 149-171

Doi: <https://doi.org/10.5569/23405104.06.02.08>

Fecha de recepción: 26-11-2017 Fecha de aceptación: 07-11-2018






\section{Introducción}

La esclerosis múltiple (EM) es una enfermedad del sistema nervioso central (SNC) caracterizada por una inflamación, desmielinización y daño axonal del cerebro y la médula espinal (Lassman, 2003), la cual afecta principalmente a la población adulta joven, normalmente entre los 20 y 40 años, y especialmente a las mujeres, en una proporción de tres a uno con respecto a los hombres (De Souza-Teixeira et al., 2009). En la actualidad, en España, podemos encontrar a 47.000 personas que sufren esta enfermedad, 600.000 en Europa y 2.500 .000 en todo el mundo (FELEM, 2017). Es una de las causas más frecuentes de la discapacidad neurológica en adultos jóvenes (Porras et al., 2007). Su curso no se puede pronosticar, y puede variar mucho de una persona a otra (FELEM, 2017), pero el $50 \%$ de los afectados es incapaz de caminar de forma autónoma a los 15 años después del inicio (Porras et al., 2007).

La etiología de la EM se desconoce en la actualidad, aunque se han implicado factores genéticos, ambientales, infecciosos y la exposición a diferentes patógenos (Giovantesnnoni y Ebers, 2007).

Esta enfermedad se puede presentar de las siguientes formas: remitente-recurrente (EMRR), forma secundaria progresiva (EMSP), forma progresiva primaria (EMPP), forma progresiva recidivante (EMPR), forma benigna (EMFB) (FELEM, 2017).

El diagnóstico se realiza a través de la historia clínica, la exploración neurológica, los potenciales evocados, la resonancia magnética nuclear (RMN) y un estudio del líquido cefalorraquídeo (FELEM, 2017), siendo la RMN la que ha demostrado más fiabilidad, dividiéndose en la RMN craneal, que detecta lesiones hasta en un $95 \%$ de los casos, y la RMN cérvico-medular, haciéndolo hasta en un $75 \%$ (Carretero et al., 2001).

Hay estudios que afirman que la EM no afecta a la duración de la vida pero, por el contrario, otros advierten que la sobrevida de estos pacientes es en promedio de 35 años tras su inicio (Kurtzke, 2000), con el pico de mortalidad entre los 55 y 64 años de edad (García-Pedroza, 2003). Se consideran variables pronósticas favorables, la edad temprana del diagnóstico, ser mujer y los síntomas de comienzo visuales y sensitivos. En cambio, el ser varón, la edad de diagnóstico superior a los 40 años, el comienzo por síntomas motores y cerebelosos, la recurrencia precoz tras el primer brote y el curso progresivo de la enfermedad, se valoran como desfavorables (Andersson et al., 1994). Sin embargo, las causas más comunes de muerte son las infecciones, enfermedades solapadas no relacionadas con la EM y el suicidio (Andersson et al., 1994).

Para un mejor pronóstico de la EM es imprescindible iniciar un tratamiento precoz, con el fin de preservar el tejido del sistema nervioso central, puesto que el cerebro de una persona con esta enfermedad se reduce entre un 0,5 y un $1 \%$ cada año, pudiendo dañar el SNC con carencia de síntomas (Arroyo, 2017).

Los síntomas dependen de las áreas del SNC lesionadas, varían entre diferentes personas y, en cada una de estas, según el momento, la gravedad y la duración, siendo estos los más comunes: fatiga; trastornos visuales; espasticidad; alteraciones de sensibilidad; trastornos del habla; problemas de vejiga e intestinales; problemas de sexualidad; trastornos cognitivos y emocionales y, los que se tratarán en profundidad en este artículo, los problemas motores, de equilibrio y coordinación, originando déficit de movilidad, pérdida de equilibrio, temblores, ataxia, vértigos y mareos, torpeza, falta de coordinación y debilidad, especialmente 
en las piernas, resultando en dificultades en la marcha (Beatty y Monson, 1996), siendo estas alteraciones motoras los síntomas más repetidos, en un $90-95 \%$ de los casos (Carretero et al., 2001).

En cuanto al perfil temporal de los síntomas, ocurren en episodios denominados brotes, recidivas o exacerbaciones, seguidas de periodos de desaparición de los mismos (Fernández et al., 2011). Frente a esto, el objetivo más importante de la investigación en EM consiste en desarrollar tratamientos que lleven a un estado NEDA ("no evidencia de actividad de la enfermedad"), buscando con ello la ausencia de brotes y el no aumento de la discapacidad. En este momento ya hay ensayos clínicos con anticuerpos monoclonales y otros tratamientos inmunomodulares para comprobar cuántos pacientes pueden estar en dicho estado durante un periodo de dos años (Arroyo, 2017).

El empleo de la realidad virtual y la 'gamificación' (el uso de técnicas, elementos y dinámicas propias de los juegos y el ocio en actividades con el fin de potenciar la motivación, reforzar la conducta para solucionar un problema, mejorar la productividad, obtener un objetivo, activar el aprendizaje y evaluar a individuos concretos) es uno de los sectores con más crecimiento de los últimos años. La investigación y el desarrollo de programas para proporcionar una ayuda en la rehabilitación motora y funcional es una de las medidas que se pretenden conseguir en el marco de la CIF (Clasificación Internacional del Funcionamiento, la Discapacidad y la Salud) (Lange et al., 2010).

Varios estudios de revisión han demostrado que una de las principales limitaciones se encuentran en el equilibrio, (Cameron y Lord, 2010), consecuencia del daño que causa esta enfermedad al cerebelo, el cual participa a través de la integración sensorio-motora en el control postural, pudiendo causar, si es dañado, déficit en el mantenimiento del equilibrio, la postura y/o el aprendizaje motor (Ayán et al., 2017). Por ello, con este estudio se pretende mostrar la importancia que puede tener la tecnología para la rehabilitación de las personas con EM, con un trabajo de equilibrio y coordinación, utilizando la plataforma NeuroAthome de realidad virtual.

\subsection{La actividad física para pacientes con esclerosis múltiple}

Históricamente numerosos autores no han mencionado la actividad física como tratamiento o ayuda para los pacientes con EM, justificándolo por la observación de que el ejercicio generaba un empeoramiento de los síntomas como alteraciones del equilibrio, problemas visuales o aumento de la espasticidad o añadiendo que evitándolo podrían preservar la energía para actividades de la vida diaria. Como consecuencia, las personas con EM han sido invitadas a tener un estilo de vida inactivo durante años (Cuevas et al., 2007). A todo ello se suma que la práctica de actividad física provoca un aumento de la temperatura corporal, dando elevaciones de la misma de 0.5 grados centígrados, lo que supone una ralentización o bloqueo de la conducción de los impulsos nerviosos (Sierra, 2010) pero, por el contrario, en estudios posteriores se ha demostrado que la práctica del ejercicio físico mejora la movilidad, control postural, fuerza y buena forma física en esta población (Thomas et al., 2014). A continuación, se presentan los problemas más importantes con los que cuentan las personas con EM y el papel que juega la actividad física.

En cuanto a la espasticidad, esta tiene una prevalencia en pacientes diagnosticados de EM de entre el 40 y el $84 \%$ en los distintos grados de gravedad (Aguilar et al., 2004) los cuales, según Vivancos et al. (2007), 
son los siguientes: fase inicial de la espasticidad (aumento de la tensión de un músculo), fase de actitud viciosa (desequilibrio muscular por predominio de la espasticidad en determinados grupos musculares), fase de retracción muscular (al persistir la actitud viciosa se produce un crecimiento desigual entre grupos musculares agonistas y antagonista), fase de deformidades osteoarticulares (se modifican las presiones).

Más concretamente en el equilibrio y coordinación, se debe atender a que más del $50 \%$ de las personas con EM sufren caídas durante un periodo de seis meses (Finlayson et al., 2006; Nilsagard et al., 2009; Matsuda et al., 2011), pudiendo requerir en ocasiones atención médica (Matsuda et al., 2011; Peterson et al., 2008). Además, el impacto se extiende más allá del evento, pues puede llevar a la restricción de actividades (Finlayson y Peterson, 2010). La importancia de las caídas es tal que las personas con EM que las han sufrido en los últimos seis meses tienen más lesiones en el cerebelo y en el tronco cerebral en comparación con los que no lo sufren (Prosperini et al., 2011). Según algunos autores, es la contribución del equilibrio la responsable entre la función cerebelosa y el riesgo de caídas (Cattaneo et al., 2002). Estas capacidades experimentan una gran mejora con la práctica de actividad física, según todos los estudios científicos que se han llevado a cabo para analizar esta capacidad, mostrándose algunos de ellos en la tabla 1.

A nivel emocional también se obtienen mejoras, puesto que otro síntoma habitual (del 8 al $55 \%$ ) es la depresión (Porras y Lilia, 2007), mostrando mejoría tras la práctica de actividad física según todos los autores que apoyan esta práctica.

Además de los beneficios ya mencionados, se encuentran resultados favorables en la fatiga y calidad de vida en personas con EM que realizan actividad física regularmente, comparándolo con los que no la realizan (Dalgas y Stenager, 2012).

\subsection{Tipos de ejercicio recomendado para personas con esclerosis múltiple}

\section{- Fuerza}

El entrenamiento de fuerza contribuye a la mejora de la repuesta cardiovascular al ejercicio, disminuyendo así la fatiga y rebajando las demandas cardiacas en la realización de cualquier actividad (Serrano, 2005). Además, se ha demostrado mejorar la capacidad de producción de fuerza, equilibrio y la calidad de vida (Kjølhede et al., 2012). Además este entrenamiento mejora la conducción neural (Dalgas et al., 2013) y la señal eferente de las neuronas motoras en sujetos con EM (Fimland et al., 2010).

Según Serrano (2005) un buen entrenamiento de fuerza en personas con EM es el entrenamiento con resistencias progresivas, tres días discontinuos a la semana, con una intensidad del $70 \% 10 \mathrm{RM}$, tres series de ocho a doce repeticiones, realizando siempre que sea posible ejercicios de una amplitud articular completa.

Otros autores, como Skjerbæk et al. (2012), han observado que los entrenamientos de fuerza de carácter progresivo con cargas submáximas tienen una gran ventaja en esta población, ya que provocan un menor aumento de la temperatura corporal en comparación con los entrenamientos aeróbicos o de resistencia, por lo que en pacientes en los que el aumento de calor suponga una pérdida de rendimiento, ánimo, etc., este tipo de entrenamiento sería el más recomendado. 


\section{- Entrenamiento aeróbico}

Uno de los problemas de esta enfermedad es que sus actividades de la vida diaria son totalmente anaeróbicas y, por lo tanto, hay más facilidad de generar fatiga. Esto ocurre porque la capacidad de prolongar un esfuerzo se ve disminuida puesto que esta patología presenta una reducción de la sección transversal del músculo donde la cantidad de fibras I es menor y estas a su vez son más pequeñas que las también deterioradas fibras II (Kent-Braun, et al. 1997). Con este entrenamiento, el consumo máximo de oxígeno puede aumentar y también intervenir en la fibra muscular aumentando las enzimas oxidativas, el número y grosor de las mitocondrias y la superficie capilar. Pero a nivel central, produce otras adaptaciones cardiovasculares que ayudan a reducir las demandas por un esfuerzo determinado. El entrenamiento aeróbico es capaz de reducir la tensión arterial y la frecuencia cardiaca por un mismo esfuerzo permitiendo prolongar una actividad en el tiempo (Serrano, 2005).

Una buena forma de trabajar esta capacidad sería empleando un método continuo, con una frecuencia de tres días a la semana, a una intensidad del 65-75\% FCMax o 50-65\% VO2Max. También sería posible mediante el método interválico, en este caso a una intensidad de 75-85\% FCMax en el periodo de mayor intensidad y de 60-80\% VO2Max, en los de una intensidad menor (Serrano, 2005).

\section{- Ejercicio en el agua}

Para trabajar en este medio, la temperatura debe estar entre 27 y $28{ }^{\circ} \mathrm{C}$ (Lesley et al., 2004). El ambiente acuático tiene propiedades únicas donde, por un lado, la flotabilidad reduce el peso corporal, que puede ayudar a mover los miembros con un rango completo de movimiento y reducir la presión sobre las articulaciones, siendo además un medio con un mínimo riesgo de lesiones. Por otro lado, la presión hidrostática en las extremidades puede ayudar a reducir la hinchazón. Respecto a la resistencia ofrecida, el agua es más densa que el aire y proporciona una mayor resistencia al movimiento, esta propiedad es importante para el entrenamiento de fuerza. De este modo, el ejercicio puede adaptarse a las necesidades de la mayoría de las personas con EM (Barbar, 2014).

\subsection{Precauciones al realizar actividad física con esclerosis múltiple}

En estos pacientes es especialmente importante mantener la temperatura corporal controlada, porque toda la sintomatología empeora con el aumento de esta y son propensos al calentamiento por un sistema nervioso autónomo deteriorado, provocando el calor un retraso de la conducción en las vías nerviosas que han perdido la mielina. Por ello, son muy importantes los medios para bajar la temperatura corporal antes, durante y después del entrenamiento. Para reducir estos problemas, el ejercicio se debería realizar exclusivamente en ambientes frescos (algunos ergómetros de piernas cuentan con un mecanismo de ventilador que puede ser un buen dispositivo para refrescar). También debería usarse una ropa apropiada (Serrano, 2005). Otras medidas son refrescar las superficies que están en contacto con el paciente y llevar un paño húmedo en el cuello o tomando un baño frío antes del ejercicio (White et al., 2000 y Petajan y White, 1999).

Por otro lado, la fatiga es un síntoma casi inherente a la enfermedad, puede ser tan incapacitante que les obligue a interrumpir toda actividad, por lo que se debe enseñar al paciente a economizar esfuerzos (Santos, 2014). 
Seguidamente, en la tabla 1, se expone una revisión bibliográfica de distintos estudios que emplean la actividad física como medio rehabilitador en pacientes con EM.

\begin{tabular}{|c|c|c|c|c|}
\hline Autores & Diseño & Muestra & Intervención & Resultados \\
\hline $\begin{array}{l}\text { Marandi et } \\
\text { al. (2013) }\end{array}$ & $\begin{array}{l}\text { - Objetivo: comparar } \\
\text { el efecto del } \\
\text { entrenamiento acuático } \\
\text { y de Pilates en el } \\
\text { equilibrio dinámico } \\
\text { - Duración: } 12 \text { semanas } \\
\end{array}$ & 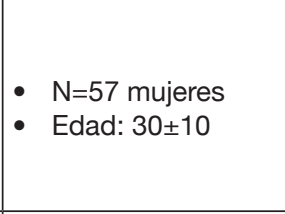 & $\begin{array}{l}\text { - Entrenamiento: } \\
\text { - Grupo A: acuático } \\
\text { - Grupo B: Pilates } \\
\text { - Frecuencia: } 3 \text { veces/ } \\
\text { - semana } \\
\text { - Duración: } 60^{\prime} \\
\end{array}$ & $\begin{array}{l}\text { Ambos grupos } \\
\text { experimentales mejoraron el } \\
\text { equilibrio dinámico. }\end{array}$ \\
\hline $\begin{array}{l}\text { Retuerta } \\
\text { (2014) }\end{array}$ & $\begin{array}{l}\text { Objetivo: comprobar } \\
\text { la efectividad de la } \\
\text { actividad física ante la } \\
\text { presencia de un brote } \\
\text { agudo. } \\
\text { - Duración: } 19 \text { semanas } \\
\text { divididas en } 3 \text { ciclos de } \\
6 \text { semanas }\end{array}$ & $\begin{array}{l}\text { - } \mathrm{N}=1 \text { hombre } \\
\text { - Edad: no } \\
\text { especificada }\end{array}$ & $\begin{array}{l}\text { - Entrenamiento primer } \\
\text { ciclo: terapia acuática+ } \\
\text { bicicleta estática } \\
\text { - Frecuencia: } 4 \text { veces/ } \\
\text { semana } \\
\text { - Duración: no } \\
\text { especificada } \\
\text { - Entrenamiento } \\
\text { segundo ciclo: terapia } \\
\text { acuática + bicicleta } \\
\text { estática + carrera } \\
\text { - Frecuencia: } 5 \text { veces/ } \\
\text { semana } \\
\text { - Duración: no } \\
\text { especificada } \\
\text { - Entrenamiento tercer } \\
\text { ciclo: terapia acuática } \\
\text { + bicicleta estática } \\
\text { +carrera } \\
\text { - Frecuencia: } 5 \text { veces/ } \\
\text { semana } \\
\text { - Duración: no } \\
\text { especificada } \\
\text { Adaptación: (2 } \\
\text { sesiones): carrera. } \\
\end{array}$ & $\begin{array}{l}\text { La espasticidad disminuyó } \\
\text { significativamente y la } \\
\text { fuerza muscular revirtió. } \\
\text { La sensibilidad y la } \\
\text { coordinación regresaron } \\
\text { a los niveles perceptivos } \\
\text { previos al brote, y la } \\
\text { fatiga pasó a ser un factor } \\
\text { controlado por el paciente. }\end{array}$ \\
\hline $\begin{array}{l}\text { Kargarfard et } \\
\text { al. (2012) }\end{array}$ & $\begin{array}{l}\text { Objetivo: examinar } \\
\text { la efectividad del } \\
\text { entrenamiento acuático } \\
\text { sobre la fatiga y la } \\
\text { calidad de vida } \\
\text { - Duración: } 8 \text { semanas }\end{array}$ & $\begin{array}{l}\text { - } \mathrm{N}=32 \text { mujeres } \\
\text { (Experimental }=10, \\
\text { control=11) } \\
\text { - Edad: } 32,6 \pm 8\end{array}$ & $\begin{array}{l}\text { - Entrenamiento: } \\
\text { acuático } \\
\text { - Frecuencia: } 3 \text { veces/ } \\
\text { semana } \\
\text { - Duración:60' }\end{array}$ & $\begin{array}{l}\text { Los pacientes del grupo } \\
\text { experimental mostraron } \\
\text { mejoras significativas en } \\
\text { fatiga y en la calidad de vida } \\
\text { comparación con el grupo } \\
\text { de control. }\end{array}$ \\
\hline $\begin{array}{l}\text { Etxebeste } \\
(2013)\end{array}$ & $\begin{array}{l}\text { - Objetivo: } \\
\text { - comprobar la } \\
\text { efectividad del ejercicio } \\
\text { físico en el tratamiento } \\
\text { y calidad de vida, } \\
\text { comprobar qué tipo } \\
\text { de ejercicio es el más } \\
\text { efectivo y conocer } \\
\text { cómo mejorar la fatiga } \\
\text { Duración: no } \\
\text { especificada }\end{array}$ & $\begin{array}{l}\text { - } \mathrm{N}=13 \text { (8 mujeres, } \\
3 \text { hombres) } \\
\text { - Edad: } 41 \pm 8\end{array}$ & $\begin{array}{l}\text { - Entrenamiento: } \\
\text { aeróbico en } \\
\text { cocloergómetro para } \\
\text { miembro inferior } \\
\text { - Frecuencia: } 8 \text { veces/ } \\
\text { semana } \\
\text { - Duración: } 40^{\prime}\end{array}$ & $\begin{array}{l}\text { Mejora de la máxima } \\
\text { tolerancia al ejercicio. } \\
\text { Cambios significativos en } \\
\text { la capacidad de la marcha. } \\
\text { No hubo diferencias } \\
\text { significativas respecto a la } \\
\text { percepción de la fatiga. La } \\
\text { diferencia percibida sobre la } \\
\text { calidad de vida fue parcial. }\end{array}$ \\
\hline
\end{tabular}




\begin{tabular}{|c|c|c|c|c|}
\hline Autores & Diseño & Muestra & Intervención & Resultados \\
\hline $\begin{array}{l}\text { Wens et al. } \\
\text { (2015) }\end{array}$ & $\begin{array}{l}\text { Objetivo: evaluar } \\
\text { las respuestas } \\
\text { contráctiles musculares } \\
\text { y la capacidad de } \\
\text { resistencia } \\
\text { - Duración: } 12 \text { semanas }\end{array}$ & $\begin{array}{ll}\text { - } & \mathrm{N}=34 \\
\text { - } & \text { Grupo } \\
& \text { sedentario=11 } \\
\text { - } & \text { Grupo } \\
& \text { experimental } 1 \\
& =12 \\
\text { - } & \text { Grupo } \\
& \text { experimental } 2 \\
& =11 \\
\text { - } & \text { Edad: no } \\
\text { especificada }\end{array}$ & $\begin{array}{l}\text { - Entrenamiento grupo } \\
\text { experimental } 1 \text { (HITR): } \\
\text { intervalo de alta } \\
\text { intensidad } \\
\text { - Entrenamiento grupo } \\
\text { experimental } 2 \\
\text { (HCTR): cardiovascular } \\
\text { continuo de alta } \\
\text { intensidad } \\
\text { - Frecuencia: no } \\
\text { especificada } \\
\text { Duración: no } \\
\text { especificada } \\
\text { Ambos en combinación } \\
\text { con entrenamiento de } \\
\text { resistencia. }\end{array}$ & $\begin{array}{l}\text { Ambos grupos } \\
\text { experimentales aumentaron } \\
\text { la fibra media CSA. La fibra } \\
\text { de CSA tipo I aumentó en el } \\
\text { grupo } 2 \text { mientras que el tipo } \\
\text { Il y la CSA aumentó en el } 1 . \\
\text { La fuerza muscular mejoró } \\
\text { en ambos y el porcentaje de } \\
\text { grasa corporal disminuyó. } \\
\text { La capacidad de resistencia } \\
\text { y masa de tejido magro } \\
\text { sólo aumentó en el grupo } \\
\text { 1. Los niveles de actividad } \\
\text { física auto-reportados } \\
\text { aumentaron en ambos }\end{array}$ \\
\hline $\begin{array}{l}\text { Barbar et al. } \\
(2014)\end{array}$ & $\begin{array}{l}\text { - Objetivo: Medir el } \\
\text { efecto del ejercicio } \\
\text { acuático en el equilibrio } \\
\text { - Duración: } 8 \text { semanas }\end{array}$ & $\begin{array}{l}\text { - } \mathrm{N}=60 \text { hombres } \\
\text { - } \quad \text { Edad: } 25 \pm 5\end{array}$ & $\begin{array}{l}\text { - Entrenamiento: } \\
\text { acuático } \\
\text { - Frecuencia: } 3 \text { veces/ } \\
\text { semana } \\
\text { - Duración: } 60^{\prime}\end{array}$ & $\begin{array}{l}\text { Aumento significativo del } \\
\text { equilibrio dinámico pero no } \\
\text { en el equilibrio estático. }\end{array}$ \\
\hline $\begin{array}{l}\text { Dettmers et } \\
\text { al. (2009) }\end{array}$ & $\begin{array}{l}\text { Objetivo: medir el } \\
\text { efecto del ejercicio en } \\
\text { la distancia máxima } \\
\text { de marcha, tiempo } \\
\text { dedicado a la marcha, } \\
\text { fatiga, depresión y } \\
\text { calidad de vida } \\
\text { - Duración: } 3 \text { semanas }\end{array}$ & $\begin{array}{l}\text { - } \mathrm{N}=30 \text { (grupo } \\
\text { control=15, grupo } \\
\text { experimental= } \\
\text { - } 15 \text { ) } \\
\text { - Edad: no } \\
\text { especificada }\end{array}$ & $\begin{array}{l}\text { - Entrenamiento grupo } \\
\text { experimental: aeróbico } \\
\text { durante } 3 \text { semanas con } \\
3 \text { veces por semana } \\
\text { de } 45 \text { minutos cada } \\
\text { sesión. } \\
\text { - Entrenamiento } \\
\text { grupo control: } \\
\text { fuerza, equilibrio y } \\
\text { coordinación } \\
\text { - Frecuencia: } 3 \text { veces/ } \\
\text { semana } \\
\text { - Duración: } 45^{\prime}\end{array}$ & $\begin{array}{l}\text { La distancia máxima } \\
\text { y tiempo dedicado a } \\
\text { la marcha aumentaron } \\
\text { significativamente en } \\
\text { el grupo experimental. } \\
\text { La fatiga mejoró } \\
\text { significativamente en grupo } \\
\text { control. La depresión y la } \\
\text { calidad de vida mejoraron } \\
\text { más en grupo control. }\end{array}$ \\
\hline $\begin{array}{l}\text { Brichetto et } \\
\text { al. (2015) }\end{array}$ & $\begin{array}{l}\text { Objetivo: evaluar } \\
\text { la eficacia de los } \\
\text { tratamientos de } \\
\text { rehabilitación en } \\
\text { trastornos del equilibrio } \\
\text { - basados en } \\
\text { deficiencias visuales, } \\
\text { somatosensoriales } \\
\text { y vestibulares } \\
\text { versus ejercicios } \\
\text { de rehabilitación } \\
\text { tradicional } \\
\text { Duración: } 4 \text { semanas }\end{array}$ & $\begin{array}{l}\text { - } \mathrm{N}=32 \\
\text { - } \text { Edad: } 50,5 \pm 11,6\end{array}$ & $\begin{array}{l}\text { Entrenamiento } \\
\text { grupo experimental } \\
\text { 1: tratamiento de } \\
\text { rehabilitación adaptado } \\
\text { a las necesidades de } \\
\text { deterioro del sistema } \\
\text { vestibular. } \\
\text { - Entrenamiento } \\
\text { grupo experimental } \\
\text { 2: tratamiento } \\
\text { estandarizado de } \\
\text { rehabilitación del } \\
\text { equilibrio. } \\
\text { Frecuencia: } 3 \text { veces/ } \\
\text { semana } \\
\text { Duración: } 60\end{array}$ & $\begin{array}{l}\text { Mejoras en el equilibrio } \\
\text { de ambos grupos pero } \\
\text { significativamente superior } \\
\text { en el grupo experimental } 1 .\end{array}$ \\
\hline
\end{tabular}




\begin{tabular}{|c|c|c|c|c|}
\hline Autores & Diseño & Muestra & Intervención & Resultados \\
\hline $\begin{array}{l}\text { Gorgas } \\
(2015)\end{array}$ & $\begin{array}{l}\text { Objetivo: Investigar los } \\
\text { efectos del equilibrio } \\
\text { basado en el peso del } \\
\text { tronco en la marcha. } \\
\text { - Duración: no } \\
\text { especificada }\end{array}$ & $\begin{array}{ll}\text { - } & \mathrm{N}=40 \\
\text { - } & \text { Edad: } 49,4 \pm \\
\text { - } & 13,4\end{array}$ & $\begin{array}{l}\text { Entrenamiento: del } \\
\text { equilibrio (caminar } \\
\text { sobre una pasarela } \\
\text { con/sin pesos ligeros) } \\
\text { - } \text { Frecuencia: no } \\
\text { especificada } \\
\text { - Duración: no } \\
\text { especificada }\end{array}$ & $\begin{array}{l}\text { Mejoras en la velocidad de } \\
\text { marcha con la aplicación de } \\
\text { pesos ligeros. }\end{array}$ \\
\hline $\begin{array}{l}\text { Burschka } \\
\text { et al. } \\
(2015)\end{array}$ & $\begin{array}{l}\text { - Objetivo: Medir el } \\
\text { efecto del Tai Chi para } \\
\text { la coordinación, el } \\
\text { equilibrio, la fatiga y } \\
\text { depresión } \\
\text { - Duración: } 24 \text { semanas }\end{array}$ & $\begin{array}{l}\text { - } \mathrm{N}=32 \text { (grupo } \\
\text { control=17, grupo } \\
\text { experimental=15 } \\
\text { - } \text { Edad: } 42,6 \pm 9,4\end{array}$ & $\begin{array}{l}\text { - Entrenamiento grupo } \\
\text { experimental: Tai Chi } \\
\text { - Entrenamiento grupo } \\
\text { control: rehabilitación } \\
\text { tradicional. } \\
\text { - Frecuencia: } 1 \mathrm{vez} / \\
\text { semana } \\
\text { - Duración: 90' }\end{array}$ & $\begin{array}{l}\text { El grupo control únicamente } \\
\text { obtuvo mejoras en el nivel } \\
\text { de fatiga, mientras que el } \\
\text { experimental mejoró en la } \\
\text { coordinación, el equilibrio y } \\
\text { la depresión. }\end{array}$ \\
\hline
\end{tabular}

Fuente: elaboración propia.

\subsection{Aplicación de las nuevas tecnologías como medio rehabilitador}

El gran avance de las nuevas tecnologías, permite la existencia de programas destinados a proporcionar una ayuda en la terapia de rehabilitación motora y funcional durante la fase de recuperación, siendo una de las medidas que se están llevando a cabo en el marco de la CIF (Lange et al., 2010). Dentro de este campo existen diferentes formatos: programas informáticos de estimulación cognitiva, realidad virtual y sistemas telemáticos. Todos ellos además de ofrecer una estimulación de distintos sistemas, también son válidos para la evaluación del paciente (Vázquez, 2013).

En nuestro trabajo es la realidad virtual la que tiene un papel principal. Esta se basa en la simulación de un entorno generado a través de una interfaz que permite al paciente la interacción con distintos elementos. Los beneficios de la realidad virtual como medio de rehabilitación son muy variados, ofreciendo un entorno seguro, una completa adaptación de los ejercicios a las capacidades del paciente, genera datos objetivos a cerca de la evolución, y, además, al plantearse como un juego la motivación aumenta en gran medida (CIEN, 2014).

Acotando el campo de atención, para centrarnos en la Plataforma NeuroAthome, aunque no se han encontrado estudios previos a cerca de su aplicación en personas con EM, pero sí ha demostrado su eficacia como parte de rehabilitación tanto física como cognitiva en otro tipo de poblaciones. Así ocurrió en uno de los estudios más recientes llevados a cabo con este instrumento, donde se tuvieron en cuenta los indicadores de equilibrio estático y dinámico y los alcances con los miembros superiores, además de valorar la percepción del usuario. Para su realización, se contó con un grupo control de once personas, al que se le aplicó un tratamiento convencional, y con otro experimental de nueve, los cuales trabajaron con el NeuroAthome. En todos los casos se llevó a cabo una sesión semanal con una duración de 45 minutos durante tres meses. Los participantes contaban con distintos tipos de enfermedades neuromusculares, tales como ELA (Esclerosis Lateral Amiotrófica), distrofias musculares y ataxias, de este modo al trabajar con diferentes patologías los resultados no son del todo concluyentes, pero sí abre puertas a futuros estudios con poblaciones más 
amplias. Los resultados de la investigación mostraron una mejora mayor en el grupo experimental, además de una mejor percepción del desarrollo de las tareas planteadas con el NeuroAthome, en comparación con el otro grupo (Olmo, 2016).

A continuación, en la tabla 2, se muestra una revisión bibliográfica de estudios donde se utilizan las nuevas tecnologías como medio rehabilitador.

\begin{tabular}{|c|c|c|c|c|}
\hline Autores & Diseño & Muestra & Intervención & Resultados \\
\hline $\begin{array}{l}\text { Prosperini } \\
\text { (2015) }\end{array}$ & $\begin{array}{l}\text { - Objetivo: Investigar si } \\
\text { un videojuego puede } \\
\text { proporcionar beneficios } \\
\text { cognitivos motores } \\
\text { - } \text { Duración: } 24 \text { semanas } \\
\end{array}$ & $\begin{array}{l}\text { - } \mathrm{N}=21 \\
\text { - } \\
\text { Edad: } \\
35,1 \pm \\
8,4\end{array}$ & $\begin{array}{l}\text { - Entrenamiento: empleo de } \\
\text { juegos de la Wii Fit Plus } \\
\text { - Frecuencia: } 5 \text { veces/ semana } \\
\text { - Duración: } 30^{\prime}\end{array}$ & $\begin{array}{l}\text { Mejora del equilibrio } \\
\text { general en ambos grupos, } \\
\text { velocidad de procesamiento } \\
\text { y atención sostenida. }\end{array}$ \\
\hline $\begin{array}{l}\text { Giampaolo et } \\
\text { al. (2013) }\end{array}$ & $\begin{array}{l}\text { - Objetivo: Evaluar la } \\
\text { diferencia en el equilibrio } \\
\text { entre un entrenamiento } \\
\text { visual de retroalimentación } \\
\text { con una Nintendo Wii y } \\
\text { Balance Board versus la } \\
\text { rehabilitación tradicional. } \\
\text { - Duración: } 4 \text { semanas }\end{array}$ & $\begin{array}{l}-\quad \mathrm{N}=36 \\
\text { - } \quad \text { Edad: } \\
43,2 \pm \\
11,5\end{array}$ & $\begin{array}{l}\text { - Entrenamiento grupo control: } \\
\text { ejercicios dinámicos sobre } \\
\text { una y dos piernas. } \\
\text { - Entrenamiento grupo } \\
\text { experimental: ejercicios } \\
\text { elegidos al azar recogidos en } \\
\text { la Nintendo, Wii y Balance. } \\
\text { - Frecuencia: } 3 \text { veces/semana } \\
\text { - Duración: } 60^{\prime}\end{array}$ & $\begin{array}{l}\text { Mejora mayor en el grupo } \\
\text { que emplea la realidad } \\
\text { virtual. }\end{array}$ \\
\hline $\begin{array}{l}\text { Ortiz et al. } \\
\text { (2013) }\end{array}$ & $\begin{array}{l}\text { - Objetivo: Demostrar las } \\
\text { posibles mejoras en el } \\
\text { equilibrio y control postural } \\
\text { entre los pacientes con } \\
\text { EM que completan un } \\
\text { entrenamiento virtual. } \\
\text { - Duración: } 10 \text { semanas }\end{array}$ & $\begin{array}{ll}\text { - } & \mathrm{N}=50 \\
& \text { Grupo } \\
\text { - } & \text { Edad: } \\
& 40 \pm 20\end{array}$ & $\begin{array}{l}\text { - Entrenamiento grupo control: } \\
\text { tratamiento de fisioterapia } \\
\text { - Frecuencia: } 2 \text { veces/semana, } \\
\text { - Duración: } 40^{\prime} \\
\text { - Entrenamiento } \\
\text { grupo experimental: } \\
\text { telerrehabilitación usando la } \\
\text { consola Xbox } 360 \text {, } \\
\text { - Frecuencia: } 4 \text { veces/semana } \\
\text { - Duración: } 20^{\prime}\end{array}$ & $\begin{array}{l}\text { Mejora respecto al equilibrio } \\
\text { general en ambos grupos. } \\
\text { Mejoras en el tiempo } \\
\text { medio de respuesta y la } \\
\text { contribución vestibular en el } \\
\text { grupo experimental. }\end{array}$ \\
\hline
\end{tabular}

Fuente: elaboración propia.

\section{Diseño metodológico}

\subsection{Objetivos}

- Objetivo general: emplear un protocolo de actuación con la plataforma NeuroAthome para analizar el resultado de un entrenamiento de equilibrio y coordinación con personas con EM.

- Objetivos específicos: comprobar la efectividad de la plataforma NeuroAthome para la mejora del equilibrio estático en las personas con EM. Analizar la evolución de la coordinación y de la amplitud del rango de movimiento de los miembros superiores e inferiores empleando la plataforma NeuroAthome en sujetos con EM. 


\subsection{Participantes}

Para realizar este trabajo ha participado un grupo experimental de cinco sujetos $(n=5)$, compuesto por cuatro mujeres y un hombre, todos ellos con EM y pertenecientes a la Asociación de Esclerosis Múltiple de Toledo (Ademto).

- Criterios de inclusión:

- Sujetos con EM diagnosticada desde hace dos años como mínimo.

- Nivel de la enfermedad que permita al sujeto ser independiente.

- Criterios de exclusión

- Presentar alguna enfermedad grave.

- Padecer algún tipo de lesión muscular, operación o fractura en el último año.

En la tabla 3 se muestra una síntesis de las características y actividad física que practica cada uno de los participantes en el estudio.

\begin{tabular}{l}
\hline Tabla 3. Características de los sujetos \\
\begin{tabular}{|l|c|c|c|c|l|}
\hline Sujeto & $\begin{array}{c}\text { Edad/ } \\
\text { género }\end{array}$ & $\begin{array}{c}\text { Edad de } \\
\text { Aparición EM }\end{array}$ & $\begin{array}{c}\text { Tipo } \\
\text { EM }\end{array}$ & Lado con más espasticidad & $\begin{array}{l}\text { ¿Realiza actividad física? } \\
\text { ¿Cuál? }\end{array}$ \\
\hline 1 & $61 / \mathrm{M}$ & 57 & EMRR & Derecho & $\begin{array}{l}\text { Sí } \\
\text { - Entrenamiento de fuerza }\end{array}$ \\
\hline 2 & $56 / \mathrm{F}$ & 28 & EMRR & Izquierdo & $\begin{array}{l}\text { Sí } \\
\text { - Entrenamiento de fuerza }\end{array}$ \\
\hline 3 & $56 / \mathrm{F}$ & 33 & EMSP & Izquierdo & $\begin{array}{l}\text { Sí } \\
\text { - Natación } \\
\text { - Estiramientos diarios con gomas }\end{array}$ \\
\hline 4 & $37 / \mathrm{F}$ & 17 & EMRR & Derecho & $\begin{array}{l}\text { Sí } \\
\text { - Entrenamiento de fuerza }\end{array}$ \\
\hline 5 & $53 / \mathrm{F}$ & 25 & EMSP & Izquierdo & $\begin{array}{l}\text { Sí } \\
\text { - Entrenamiento de fuerza }\end{array}$ \\
\hline
\end{tabular}
\end{tabular}

Fuente: elaboración propia.

\subsection{Variables}

- Variables independientes:

- Programa realidad virtual con NeuroAthome.

- Escala de equilibrio de Berg.

- Variable dependiente:

- Equilibrio.

- Coordinación. 


\subsection{Instrumentos}

- $\quad$ Test del equilibrio de Berg: Se trata de una escala desarrollada por primera vez en 1989 resultando un test muy funcional puesto que sus 14 pruebas se basan en ejercicios que forman parte de la vida diaria de una persona, siendo necesario poder mantenerse en bipedestación para su ejecución. La totalidad de las tareas están valoradas en una escala del 0 al 4, calificándose algunas de ellas conforme a la calidad de la ejecución, y otras según el tiempo necesario para completarla. Una vez finalizado, es posible alcanzar una puntuación máxima de 56, lo que significaría tener un excelente equilibrio.

En relación a este test, es necesario para llevar a cabo las diferentes pruebas la utilización de dos sillas, un escalón, una cinta métrica y un cronómetro.

- Kinect: sensor presentado como accesorio de la consola de videojuegos de Xbox 360, posibilitando el reconocimiento de los movimientos del paciente sin ningún contacto físico. Para su funcionamiento emplea una interfaz natural reconociendo los gestos, comandos de voz y objetos.

- Tablet Surface Pro 4: tableta independiente desde la que se ha manejado el entrenamiento.

- Plataforma Neuroathome: se trata de una plataforma software de rehabilitación virtual, diseñada con el fin de tratar secuelas tanto de una lesión neurológica como de una enfermedad neurodegenerativa, adaptándose a cualquier nivel. El tipo de rehabilitación que ofrece puede ser tanto física como cognitiva, estando constituidos por 40 ejercicios cada uno de estos tipos.

Esta plataforma además ofrece información muy detallada una vez realizado el entrenamiento, pudiendo ver de forma muy sencilla y clara los efectos de los ejercicios en cada usuario, para lo cual se generan los siguientes tipos de informes:

- Informes globales: este indicador muestra los datos de las tareas realizadas por los pacientes en dos fechas concretas, pudiendo analizar de una forma cómoda y rápida las deferencias y evolución existentes.

- Informes por tarea: en este caso el software proporciona un análisis global de cada tarea, mostrando la evolución del paciente respecto al nivel, repeticiones y tiempo empleado en la ejercicios de cada tarea, además de añadir una comparación entre las tres primeras y últimas sesiones, mostrando de una manera gráfica todas las variables mencionadas.

- Informes por fecha: se trata de otra manera con la que se pueden consultar todos los datos obtenidos de los ejercicios, mostrando las diferencias en cada uno de los días.

Más adelante de este trabajo se puede observar un ejemplo de los informes globales y por tarea (figura 1,2 y 3) que han generado los sujetos sometidos al entrenamiento.

\subsection{Procedimiento}

Se ha llevado a cabo un estudio experimental, donde se ha comenzado con una búsqueda de sujetos, teniendo una primera fase de familiarización con el NeuroAthome, previa a la toma de datos, observándose las características y limitaciones de cada uno de ellos, con el fin de ajustar los niveles de los ejercicios de entrenamiento. La semana siguiente se ha realizado el pre test, utilizando para ello la escala del equilibrio de 
Berg, para dar comienzo en los días siguientes al entrenamiento de seis semanas, el cual se ha compuesto de tres bloques de tres ejercicios cada uno de ellos, con una duración total de 20 minutos.

\subsection{Valoración}

La valoración del entrenamiento se ha realizado mediante los informes generados por el NeuroAthome, ofreciendo las gráficas de equilibrio, aciertos, fallos, tiempo requerido para el desempeño de la tarea y nivel de cada uno de los ejercicios, además de una gráfica generada al final del entrenamiento donde se muestra la evolución del paciente. Una vez finalizado este, se ha procedido a efectuar el post test, utilizando la misma metodología empleada en el pre test para poder establecer comparaciones con este.

\section{Resultados}

\subsection{Análisis cuantitativo}

Analizando la tabla que se expone a continuación (tabla 4), donde se presentan los resultados de la prueba no paramétrica, se aprecia que todos los valores tienden hacia una diferencia significativa pero únicamente en el caso del par 12 y 13 la alcanzan.

\begin{tabular}{|c|c|c|}
\hline & z (Wilconxon) & p-valor \\
\hline Par 1 & -1 & 0,317 \\
\hline Par 2 & $-1,414$ & 0,157 \\
\hline Par 3 & 0 & 1 \\
\hline Par 4 & -1 & 0,317 \\
\hline Par 5 & $-1,732$ & 0,083 \\
\hline Par 6 & -1 & 0,317 \\
\hline Par 7 & $-1,414$ & 0,157 \\
\hline Par 8 & -1 & 0,317 \\
\hline Par 9 & $-1,732$ & 0,083 \\
\hline Par 10 & -1 & 0,317 \\
\hline Par 11 & $-1,414$ & 0,157 \\
\hline Par 12 & -2 & 0,046 \\
\hline Par 13 & -2 & 0,046 \\
\hline Par 14 & $-1,732$ & 0,083 \\
\hline
\end{tabular}

Fuente: elaboración propia a partir del análisis estadístico SPSS. 
sA continuación, en la tabla 5, se muestran los resultados obtenidos en el pre y post en la prueba del equilibrio de Berg. En el caso de la media, tanto en el pre como en el post se alcanza el valor máximo (4) obteniéndolo además en la misma prueba. Distinto es el caso del valor mínimo en la misma variable, puesto que en el caso del pre se trata de 1,200 en la prueba 14 y 1,800 en el post de la misma prueba.

\begin{tabular}{|c|c|c|c|c|c|}
\hline & $\mathbf{N}$ & Media & $\begin{array}{l}\text { Desviación } \\
\text { estándar }\end{array}$ & Mínimo & Máximo \\
\hline P. 1 Pre & 5 & 3,600 & 0,547 & 3 & 4 \\
\hline P. 2 Pre & 5 & 3,400 & 0,894 & 2 & 4 \\
\hline P. 3 Pre & 5 & 4,000 & 0 & 4 & 4 \\
\hline P. 4 Pre & 5 & 3,600 & 0,547 & 3 & 4 \\
\hline P. 5 Pre & 5 & 3,200 & 0,836 & 2 & 4 \\
\hline P. 6 Pre & 5 & 3,400 & 0,894 & 2 & 4 \\
\hline P. 7 Pre & 5 & 2,400 & 1,816 & 0 & 4 \\
\hline P. 8 Pre & 5 & 2,800 & 1,303 & 1 & 4 \\
\hline P. 9 Pre & 5 & 3,200 & 0,447 & 3 & 4 \\
\hline P. 10 Pre & 5 & 3,400 & 0,894 & 2 & 4 \\
\hline P. 11 Pre & 5 & 3,000 & 0,707 & 2 & 4 \\
\hline P. 12 Pre & 5 & 2,200 & 1,303 & 0 & 3 \\
\hline P. 13 Pre & 5 & 2,000 & 1,224 & 0 & 3 \\
\hline P. 14 Pre & 5 & 1,200 & 0,836 & 0 & 2 \\
\hline P. 1 Post & 5 & 3,800 & 0,447 & 3 & 4 \\
\hline P. 2 Post & 5 & 3,800 & 0,447 & 3 & 4 \\
\hline P. 3 Post & 5 & 4,000 & 0 & 4 & 4 \\
\hline P. 4 Post & 5 & 3,800 & 0,447 & 3 & 4 \\
\hline P. 5 Post & 5 & 3,800 & 0,447 & 3 & 4 \\
\hline P. 6 Post & 5 & 3,600 & 0,547 & 3 & 4 \\
\hline P. 7 Post & 5 & 2,800 & 1,643 & 1 & 4 \\
\hline P. 8 Post & 5 & 3,000 & 1,414 & 1 & 4 \\
\hline P. 9 Post & 5 & 3,800 & 0,447 & 3 & 4 \\
\hline P. 10 Post & 5 & 3,600 & 0,547 & 3 & 4 \\
\hline P. 11 Post & 5 & 3,400 & 0,894 & 2 & 4 \\
\hline P. 12 Post & 5 & 3,000 & 1,224 & 1 & 4 \\
\hline P. 13 Post & 5 & 2,800 & 1,303 & 1 & 4 \\
\hline P. 14 Post & 5 & 1,800 & 1,095 & 0 & 3 \\
\hline
\end{tabular}

Fuente: elaboración propia a partir del análisis estadístico SPSS. 


\subsection{Análisis cualitativo}

A continuación se muestran algunos de los resultados obtenidos en el conjunto de todas las pruebas realizadas, exponiendo en primer lugar la diferencia entre el límite de estabilidad (donde el paciente tendrá menos riesgo de caída, y por tanto mejor equilibrio cuanto más amplitud tenga la gráfica), la velocidad de movimiento y de inclinación lateral de la cabeza que se ha producido entre la primera y la última sesión del entrenamiento. Seguidamente se presentan dos tablas adicionales en las que se pueden observar el rango de movimiento y la velocidad máxima del miembro inferior y superior respectivamente, al igual que en el caso de la primera, ambas comprenden las seis semanas de duración del estudio, comparándose los datos del principio con el final del entrenamiento. En todos los casos, los resultados se muestran en dos colores, atribuyendo el color rojo a la primera sesión, y el gris a la fecha de finalización.

\subsection{Informes globales}

\section{- $\quad$ Sujeto 2}

En el informe global del sujeto 2 (figura 1) se aprecia una clara diferencia en el límite de estabilidad al comienzo y al final del estudio, siendo esta muy inferior el primer día de entrenamiento, puesto que se observa un desplazamiento en todas las direcciones, reduciéndose notablemente en la finalización de este. En el caso de la velocidad de movimiento y de inclinación de la cabeza, ocurre algo similar, puesto que el primer día, se tiende a un desplazamiento hacia ambos lados, dispersándose el día de finalización hacia arriba y abajo.

\section{Figura 1. Representación del equilibrio general del sujeto 2}

\begin{tabular}{|c|c|c|}
\hline Límite de estabilidad & $\begin{array}{c}\text { Velocidad de movimiento } \\
(\mathrm{m} / \mathrm{s})\end{array}$ & $\begin{array}{c}\text { Velocidad de cabeza } \\
\text { (m/s) }\end{array}$ \\
\hline & &
\end{tabular}

Fuente: elaboración propia a partir de los informes generados por el NeuroAthome. 
En el análisis del miembro inferior (figura 2), en el caso de la pierna izquierda todos los datos referentes a la abducción y aducción han aumentado en la última sesión respecto a la primera, hecho que no se extiende a la flexión y extensión, donde hay más disparidad de resultados. Esto último es lo que sucede en la abducción y aducción del lado derecho, en cambio, en los datos obtenidos de la flexión y extensión todos los valores sufren un descenso, a excepción de las repeticiones de flexión.

Figura 2. Informe global del miembro inferior del sujeto 2 incluyendo las representaciones del rango de movimiento de la cadera y la velocidad máxima de movimiento de los pies

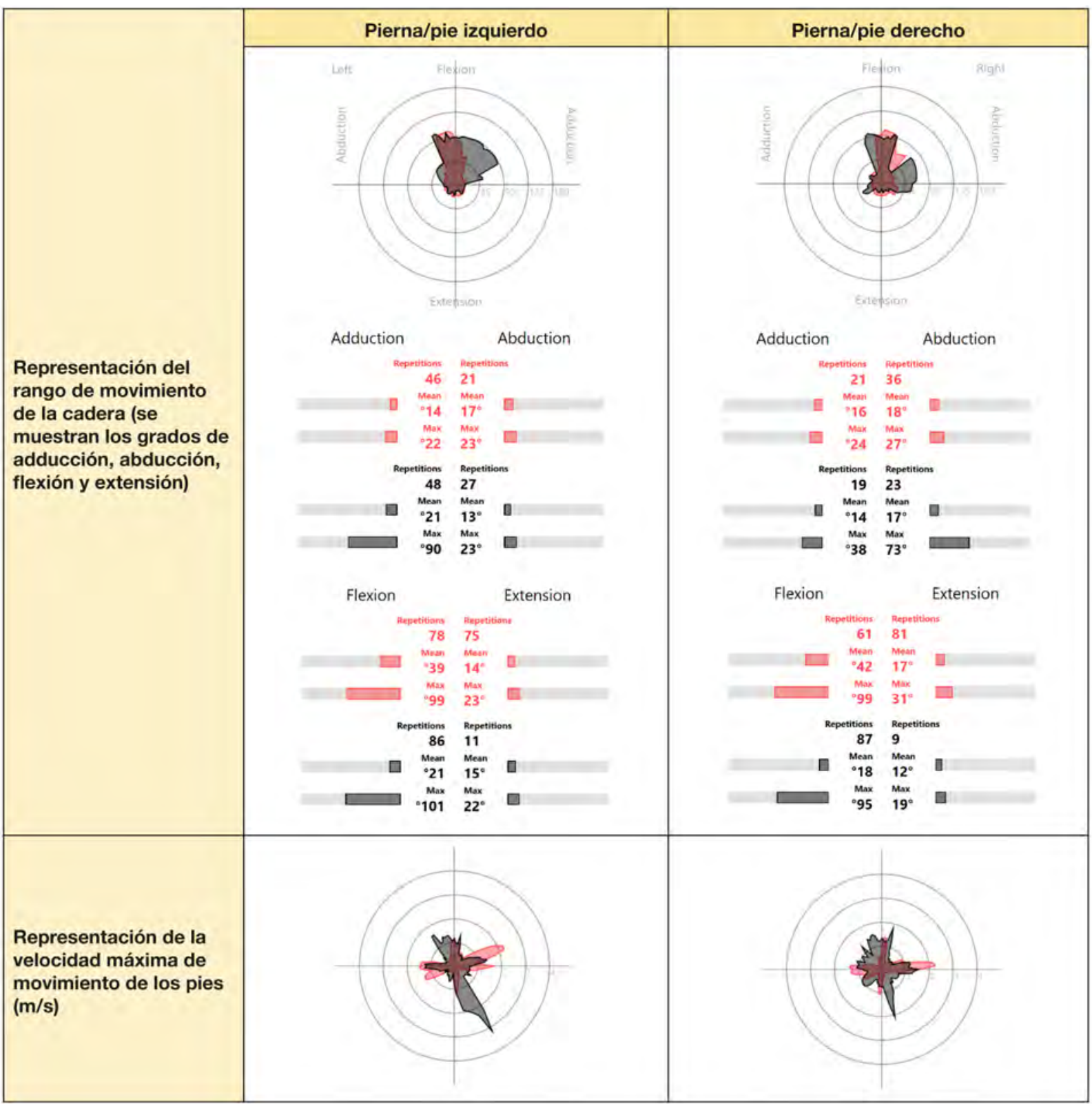

Fuente: elaboración propia a partir de los informes generados por el NeuroAthome. 
En el informe global del miembro superior del sujeto 2 (figura 3), en todos los datos obtenidos acerca del lado izquierdo se pueden encontrar tanto aumentos como disminuciones en las cifras en un porcentaje muy similar. Ligeramente distinto es el caso de la pierna derecha, donde al observar todas las variables se puede comprobar mayor número de factores que aumentan su grado. Hay que destacar por otro lado la velocidad de movimiento del lado izquierdo, donde en la primera sesión hay una concentrada tendencia hacia valores negativos tanto en el eje de ordenadas como abscisas.

Figura 3. Informe global del miembro superior sujeto 2 incluyendo las representaciones del rango de movimiento de los hombros y la velocidad máxima de movimiento de las manos

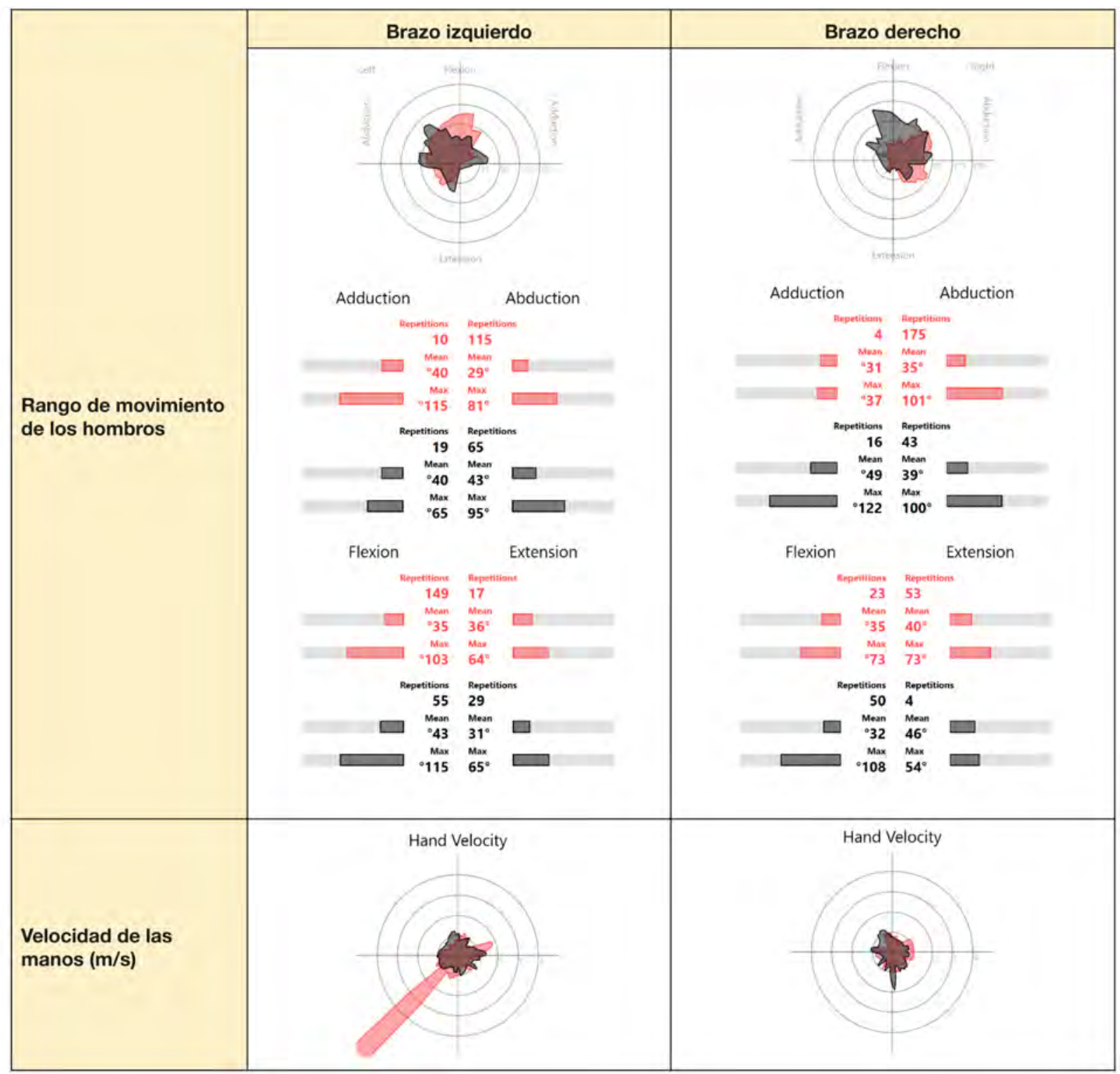

Fuente: elaboración propia a partir de los informes generados por el NeuroAthome. 


\subsection{Informes prueba más significativa: equilibrio estático lateral}

En la figura 5 se han recopilado los informes por tarea de todos los participantes referentes a una de las pruebas más significativas del test que ha pasado tanto al comienzo como al final. Haciendo una comparación entre las tres primeras y últimas sesiones del entrenamiento, en la mayoría de los casos se puede observar como hay un aumento del nivel y las repeticiones, o en su defecto, de una de estas, a excepción del sujeto número 4.

Figura 5. Informe de cada sujeto referente a un ejercicio del programa de entrenamiento de Neuroathome que mide el equilibrio estático lateral

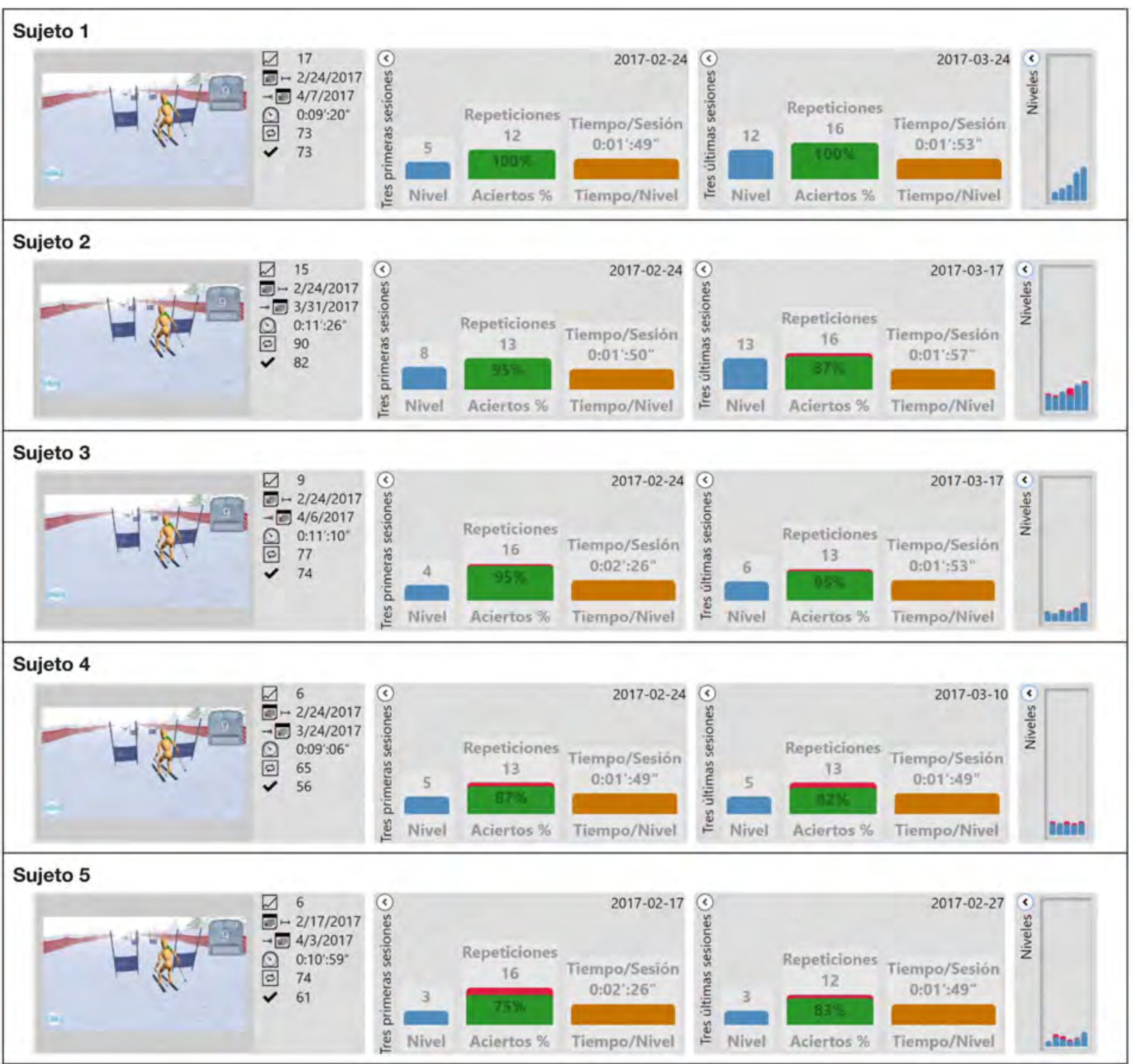

Fuente: elaboración propia a partir de los informes generados por el NeuroAthome. 


\section{Discusión}

Los resultados obtenidos en este estudio de entrenamiento del equilibrio y coordinación empleando la plataforma NeuroAthome, donde se han tenido en cuenta los datos de equilibrio estático, coordinación y rango de movimiento, muestran que todos los sujetos que han participado han tenido mejoras en diferentes variables.

Lo expuesto anteriormente queda reflejado en el análisis cuantitativo con el empleo del Test del Equilibrio de Berg, obteniendo como se puede apreciar en la tabla 4 una diferencia significativa en las pruebas número 12 y 13. Por el contrario, aunque solo se hayan obtenido estas diferencias en dichas pruebas, son muy importantes puesto que este test lo conforman una totalidad de 14 tareas, siendo estas de dificultad creciente a medida que se avanza en ellas. Siguiendo con la misma tabla, se observa que en todos los demás ítems también hay mejora, aunque esta no se considere significativa. Este hecho se manifiesta igualmente en la tabla siguiente, puesto que los valores de la media resultantes del test del Equilibrio de Berg, conformado por pruebas valoradas del 0 al 4, pasan de ser 2,957 en el caso del pre a 3,357 en el post. La razón de que muchas de las diferencias no sean significativas probablemente se deba a la corta duración del entrenamiento o el bajo número de la muestra. Esto queda reflejado en la tabla 1, donde omitiendo el estudio de Gorgas, (2015), puesto que únicamente consistió en llevar a cabo dos ensayos, atendiendo a los demás estudios el número medio de semanas en las que se ha llevado a cabo un programa de actividad física para personas con EM es de 29 sesiones, estando estas en algunas ocasiones muy concentradas, como es el caso del estudio de Retuerta, (2014), donde se llegan a realizar hasta cinco sesiones por semana, y siendo 24 el número de sesiones de entrenamiento que más se repite, conformando casi la mitad de la revisión bibliográfica que se ha realizado, con los estudios de Kargarfard et al. (2012), Etxebeste, (2013), Barbar et al. (2014) y Burschka et al. (2016).

Por otro lado, analizando la misma tabla, se comprueba que el número medio de sujetos que han participado en los estudios es de 36, a excepción de la investigación de Retuerta, (2014), donde se estudia en profundidad únicamente a un participante, por lo que la cifra de sujetos con los que suelen contar estos estudios es muy superior a la de este trabajo, donde únicamente tenemos cinco participantes.

No se han encontrado estudios dedicados a la rehabilitación de personas con EM empleando la plataforma NeuroAthome, por lo que todas las siguientes comparaciones se realizarán guiándonos por entrenamientos utilizando otros modelos de realidad virtual en esta población que, aunque también son escasos, contamos con algunos de ellos.

En nuestro estudio, en la figura 5 se aprecia como el sujeto que ha obtenido más mejoras en la prueba del equilibrio estático lateral (tarea que más se identifica con el test que se ha pasado), la cual trata de cambiar el peso corporal de lado, aspecto cuya importancia fue medida en el estudio de Gorgas (2015), resultando tener una transferencia directa a la mejora y estabilidad de la marcha, ha sido el participante número 1, siendo este el único hombre, con la consiguiente contradicción de la afirmación de García-Pedroza (2003) respecto a que ser del género masculino es un factor negativo en la $E M$, pero hay que añadir que se trata del sujeto que lleva menos número de años con la enfermedad, no siendo este un factor relevante, puesto que el único participante que no experimenta mejoras en dicha tarea es el número 4 el cual, después del an- 
terior, es el que lleva menos tiempo con la enfermedad, pudiendo comprobar que en cuanto a la progresión de la EM no existen dos pacientes iguales (Arroyo, 2017) aunque en la actualidad ambos convivan con el mismo tipo de EM, siendo esta del tipo EMRR. Todos los demás, en mayor o menor medida, han alcanzado progresos en los valores obtenidos en dicha prueba al final del entrenamiento con respecto al comienzo del mismo. En el trabajo de Brichetto et al. (2015), utilizando la plataforma Wii se obtuvieron grandes mejoras en variables como el equilibrio estático y dinámico, como ha sido nuestro caso, entre otros. Ortiz et al. (2013), utilizaron la Xbox 360, alcanzando mejoras en el equilibrio general. Otros estudiosos como Prosperini et al. (2013) han afirmado la eficacia en el entrenamiento con el tablero de balance Wii para mejorar el equilibrio estático y dinámico, entre otros.

Por otro lado, respecto a los dos sujetos con una EMSP, la cual se da cuando el grado de discapacidad aumenta, en el análisis por tarea del equilibrio dinámico general (figura 5), se muestra como en el caso del participante número 3 aumenta el nivel alcanzado aunque manteniendo idéntico el número de aciertos, en cambio, en el sujeto número 5 se mantiene el nivel inicial pero incrementando las repeticiones correctas, de esta manera se comprueba un progreso, aunque diferente, existente en los dos casos. Pero hay que añadir que el sujeto número 5 emplea una silla de ruedas para desempeñar tareas de su vida diaria, y cuenta con un grado mayor de espasticidad, sin embargo la actividad física que realiza es mucho mayor y variada al otro participante. En este sentido queda comprobado que la práctica de actividad física, mejora el grado de movilidad y control postural entre otros, de las personas con EM (Thomas et al. 2014).

Para la mejora del equilibrio, el control postural y la coordinación tienen un papel esencial, como afirman Ortiz et al. (2013), añadiendo que ambas son determinantes para la mejora del primero. Así, todos los sujetos que han tenido una mejora en la tarea de equilibrio estático lateral, también han manifestado mejoras en el límite máximo de velocidad, velocidad máxima de movimiento y de la cabeza. Respecto a este último aspecto, no se han encontrado estudios donde se mida la velocidad de la cabeza y su implicación en el equilibrio y coordinación. Sin embargo en este trabajo todos los sujetos han podido alcanzar una mayor velocidad de esta al finalizar el entrenamiento, lo que ha contribuido a la mejora del equilibrio puesto que cuanto más rápidamente se ejecuten estos movimientos, los reflejos del cuello pueden llevar a cabo los ajustes necesarios del cuerpo para mantenerlo alineado con la cabeza (Gowitzke y Milner, 1999).

Por último, en la mayoría de los sujetos ha mejorado la velocidad máxima tanto de las piernas como de las manos lo que, según Burschka et al. (2015), resulta en un progreso directo en la coordinación, pero este incremento no se ha producido en todas las direcciones, en todos los casos ha sido únicamente hacia delante y hacia detrás pero hasta el momento no se han encontrado artículos que diferencien los progresos en las direcciones de dichas mejoras. No ha sido posible encontrar ningún estudio científico acerca de la coordinación en esta población mediante el empleo de la realidad virtual, el único documento que se ha localizado a cerca de la incidencia de la actividad física en dicha variable es el de Burschka et al. (2015), donde el grupo experimental tuvo mejoras en este aspecto mediante la práctica de tai chi.

En cuanto a la distinción entre los lados con los que cada participante cuenta con mayor espasticidad, no ha habido distinción en las mejoras de la velocidad tanto del miembro superior como inferior, mejorando los dos lados y quedando demostrado que el trabajo de equilibrio y coordinación puede mejorar la espasticidad tanto del lado de mayor como de menor afectación del cuerpo, como queda expuesto en el artículo científico de Vivancos, et al. (2007). 
Por último, analizando los grados de movimiento alcanzados, en todos los casos salvo en el primer sujeto que ha progresado en el lado derecho e izquierdo de igual manera, se han encontrado mayores mejoras en el lado con menor espasticidad. En el sujeto 5 se ha alcanzado mayor grado de amplitud de movimiento en la primera sesión que en la última. La espasticidad fue evaluada por Retuerta, (2014) llevando a cabo un entrenamiento de terapia acuática, bicicleta estática y carrera a pie, con el resultado de una mejora de esta variable entre otras.

\section{Conclusiones}

Tras realizar el estudio se pueden obtener las siguientes conclusiones.

- Se afirma la eficacia en del uso de la plataforma NeuroAthome, llevando a cabo un entrenamiento del equilibrio y coordinación en la rehabilitación física de las personas con EM.

- La plataforma NeuroAthome constituye un elemento de gran utilidad en la mejora de equilibrio estático.

- Dicha plataforma también es capaz de mejorar la coordinación y aumentar los rangos de movimiento tanto de las extremidades superiores como inferiores llevando a cabo un entrenamiento del equilibrio y coordinación.

\section{Limitaciones}

- Problemas de salud y personales de los participantes: obstáculos provocados en su mayoría como consecuencia de la EM (brotes, depresión, cambios de temperatura ambiental y circunstancias personales), y dando lugar a que alguna de las sesiones no se pudiera realizar de la mejor manera posible.

- Bajo número de la muestra: existiendo además dos abandonos durante los entrenamientos en los que se ha llevado a cabo el trabajo.

- Limitación bibliográfica: ha resultado muy complicado encontrar investigación acerca de entrenamiento y efectos empleando el NeuroAthome, debido a tratarse de una plataforma muy novedosa. 


\section{Referencias bibliográficas}

Aguilar, M. et al. (2004): "Guía terapéutica de la espasticidad del adulto con toxina botulínica". Rev Neurol 38 : 971-978.

Andersson, M. et al. (1994): "Cerebrospinal fluid in the diagnosis of multiple sclerosis: a consensus report". $J$ Neurol Neurosurg Psychiatry, 57(8): 897-902.

Arroyo, R. (2017): El tiempo en esclerosis múltiple, una carrera a contrarreloj (en línea). <http://www.efesalud.com/ noticias/tiempo-en-esclerosis-multiple/>, acceso 16 de marzo de 2017.

Ayán, C. L. et al. (2017): "Esclerosis múltiple y ejercicio físico: revisión bibliográfica, situación actual y perspectivas de futuro". Medicina de l'esport, 144 : 17-26.

Barbar, A. et al. (2014): "The effect of aquatic exercise on balance of adults with multiple sclerosis". European Journal of Experimental Biology, 4 (1):38-43.

Beatty, W.W. y Monson, N. (1996): "Problem solving by patients with multiple sclerosis: Comparison of performance on the Wisconsin and California Card Sorting Tests". Journal of International Neuropsychological Society, 2: 134-140.

Brichetto, G. et al. (2015): "Tailored balance exercises on people with multiple sclerosis: A pilot randomized, controlled study". Journal Mult, 21 (8):1055-1063.

Burschka, J. M. et al. (2015): "Mindfulness-based interventions in multiple sclerosis: beneficial effects of Tai Chi on balance, coordination, fatigue and depression”. BMC Neurol 14:165 (en línea). <https://doi.org/10.1186/ s12883-014-0165-4>, acceso 12 de diciembre de 2018.

Cameron, M. y Lord, S. (2010): "Postural Control in Multiple Sclerosis:Implications for Fall Prevention". Curr Neurol Neurosci Rep, 10: 407-412.

Carretero, J. L. et al. (2001): Actualización: esclerosis múltiple. Madrid: editorial Medifam.

Cattaneo, D. et al. (2002): "Risks of falls in subjects with multiple sclerosis". Arch Phys Med Rehabil, 83: 864-867.

Centro especial de enfermedades neurológicas CIEN (2014): Rehabilitación con realidad virtual, una herramienta innovadora en la fisioterapia (en línea). <http://emalbacete.es/realidad-virtual-y-rehabilitacion/>, acceso 1 de mayo de 2017.

Cuevas, C. et al. (2007): "Consenso Mexicano para la Esclerosis Múltiple. Guía diagnóstica y terapéutica". Rev Mex Neuroci 8 (2): 155-162.

Dalgas, U. et al. (2013): "Neural drive increases following resistance training in patients with multiple sclerosis". Journal of neurology, 260 (7): 1822-1832.

Dalgas, U. y Stenager, E. (2012): "Exercise and disease progression in multiple sclerosis: can exercise slow down the progression of multiple sclerosis?". National Library of Medicine National Institutes of Health, 5 (2): 81-95.

De Souza-Teixeira, F. et al. (2009): "Effects of Resistance Training in Multiple Sclerosis". International Journal of Sports Medicine, 30: 245-250.

Dettmers, C. et al. (2009): "Endurance exercise improves walking distance in MS patients with fatigue". Acta Neurol Scand, 120 (4):251-257. 
Etxebeste, A. (2013): "Ejercicio físico en pacientes con esclerosis múltiple” (Trabajo de Grado). Escuela Universitaria de Estudios Sanitarios. Universidad pública de Navarra.

Federación Española para la Lucha contra la Esclerosis Múltiple. FELEM (2017) (en línea). <http://www.esclerosismultiple.com/>, acceso 19 de febrero de 2017.

Fernández, O. et al. (2011): Esclerosis Múltiple. Edit. Momento médico.

Fimland, M. S. et al. (2010): "Enhanced neural drive after maximal strength training in multiple sclerosis patients". Europeanjournal of appliedphysiology, 110 (2): 435-443.

Finlayson, M. L. y Peterson, E.W. (2010): “Falls, aging, and disability”. Phys Med Rehabil Clin N Am, 21: 357-373.

Finlayson, M. L. et al. (2006): "Risk factors for falling among people aged 45 to 90 years with multiple sclerosis". Arch Phys MedRehabil, 87: 1274-1279.

García-Pedroza, F. (2003): Neuroepidemiología de la esclerosis múltiple. El manejo integral de la esclerosis múltiple. México: Editorial Prado.

Giampaolo, B. et al. (2013): "The effect of Nintendo® Wii® on balance in people with multiple sclerosis: a pilot randomized control study". Multiple Sclerosis Journal, 19 (9): 1219-1221.

Giovantesnnoni, G. y Ebers, G. (2007): "Múltiple sclerosis: the environment and causation”. Curr Opin Neurol, 20 : 261-268.

Gorgas, A. M. et al. (2015): "Gait Changes with Balance-Based Torso-Weighting in People with Multiple Sclerosis". $P M C$ (en línea). <https://doi.org/10.1002/pri.1595>, acceso 12 de diciembre de 2018.

Gowitzke, B. y Milner, M. (1999): El cuerpo y sus movimientos. Bases científicas (en línea). <http://www.paidotribo. com/ficha.aspx?cod=00451>, acceso 5 de mayo de 2017.

Kargarfard, M. et al. (2012): "Effect of Aquatic Exercise Training on Fatigue and Health-Related Quality of Life in Patients with Multiple Sclerosis". NCBI (10):1701-1708.

Kent-Braun, J. A. et al. (1997): "Strength, skeletal muscle composition, and enzyme activity in multiple sclerosis". J Appl Physiol, 83: 1998-2004.

Kjølhede, T. et al. (2012): "Multiple sclerosis and progressive resistance training: a systematic review. Multiple". Sclerosis Journal 18(9):1215-1228.

Kurtzke, F. J. (2000): "Multiple sclerosis in time and space--geographic clues to cause". US National Library of Medicine National Institutes of Health. NCBI 2:S134-140.

Lange, B. S. et al. (2010): "The potential of virtual reality and gaming to assist successful aging with disability". Phys Med Rehabil Clin Am, 21: 339-356.

Lassmann, H., (2003): “Axonal injury in multiple sclerosis”. J Neurol Neurosurg Psychiatry, 74: 695-697.

Lesley, J. et al. (2004): "Exercise and Multiple Sclerosis”. Sports Med, 34 (15): 1077-1100.

Marandi, S. M. et al. (2013): "A comparison of 12 weeks of pilates and aquatic training on the dynamic balance of women with mulitple sclerosis". J Prev Med 4 (1): S110-7.

Matsuda, P. N. et al. (2011): "Falls in multiple sclerosis". PM R, 3: 624-632.

Nilsagard, Y. et al. (2009): "Predicting accidental falls in people with multiple sclerosis a longitudinal study". Clin Rehabil 23: 259-269. 
Olmo, E. (2016): Neuroathome en el tratamiento de enfermedades neuromusculares (en línea). <http://rteinsas. wixsite.com/mundortein/single-post/2016/12/09/NEUROATHOME-EN-EL-TRATAMIENTO-DE-ENFERMEDADES-NEUROMUSCULARES>, acceso 1 de junio de 2017.

Ortiz, R. et al. (2013): "A telerehabilitation program improves postural control in multiple sclerosis patients: a spanish preliminary study". J. Environ. Res. Public Health, 10: 5697-5710.

Petajan, H. J. y White, T. A. (1999): "Recommendations for physical activity in patiens with multiple sclerosis". Sport medicine, 3: 179-191.29

Peterson, E. W. et al. (2008): "Injurious falls among middle aged and older adults with multiple sclerosis". Med Rehabil 89: 1031-1037.

Porras, M. et al. (2007): “Esclerosis múltiple”. Rev Mex Neuroci, 8 (1): 57-66.

Porras, M. y Lilia, N. (2007): "Evaluación de los pacientes con esclerosis múltiple recurrente-remitente en tratamiento con interferón beta 1-a de 12 millones de unidades". Revista Mexicana de Neurociencia, 8 (1): 8-17.

Prosperini, L. et al. (2015): "Far transfer effect associated with video game balance training in multiple sclerosis: from balance to cognition?". J Neurol, 262: 774-776.

Prosperini, L. et al. (2013): "Home-based balance training using the wii balance board: A randomized, crossover pilot study in multiple sclerosis". Neurorehabilitation and Neural. 27 (6): 516-525.

Prosperini, L. et al (2011): "Therelationship between infratentorial lesions, balance deficit and accidental falls in multiple sclerosis". J Neurol Sci, 304: 55-60.

Retuerta, M. (2014): “Esclerosis múltiple y ejercicio físico tras brote agudo. Caso clínico”. Reduca (Enfermería, Fisioterapia y Podología), 6 (3): 235-261, 2014.

Santos C. (2014). Efectividad de la hidrocinesiterapia en esclerosis múltiple: revisión sistemática (tesis de grado). Facultad de Ciencias de la Salud. Universidad de Jaén.

Serrano, J. (2005): “Tratamiento fisioterapéutico de la fatiga en esclerosis múltiple”. Elselvier, 27 (4):219-27.

Sierra, M. B. (2010): Ejercicio físico en personas con esclerosis múltiple (en línea) <http://www.efdeportes.com/ efd140/ejercicio-fisico-en-personas-con-esclerosis-multiple.htm>, acceso 16 de mayo de 2017.

Skjerbæk, A. G. et al. (2013): "Heat sensitive persons with multiple sclerosis are more tolerant to resistance exercise than to endurance exercise". Mult Scler, 19 (7): 932-940.

Thomas, S. et al. (2014): "Testing the feasibility and acceptability of using the Nintendo Wii in the home to increase activity levels, vitality and well-being in people with multiple sclerosis: protocol for a pilot randomised controlled stud". BMJ Open, 4:e005172.

Vázquez, L. (2013): “Uso de las nuevas tecnologías en la intervención neuropsicológica en demencias”. Rev psicología.com, 17:14.

Vivancos, F. et al. (2007): "Guide to the comprehensive treatment of spasticity”. Rev Neurol, 45 (6):365-375.

Wens, I. (2015): “iHigh Intensity Exercise in Multiple Sclerosis: Effects on Muscle Contractile Characteristics and Exercise Capacity, a Randomised Controlled Trial”. Plos one, 10 (9).

White, A. T. et al. (2000): "Effect of precooling on physical performance in multiple sclerosis". Mult Scler, 6: 176-80. 